\title{
Algunas consecuencias filosóficas del trabajo de Kurt Gödel*
}

\author{
Carlos Alberto Cardona SuÁrez \\ Departamento de Ciencias Básicas \\ Universidad Jorge Tadeo Lozano \\ carcardona@sky.net.co
}

\begin{abstract}
Resumen: El artículo presenta los argumentos que le permiten a Gödel defender el realismo platónico a partir de sus resultados de incompletitud. Se sostiene que hay un estrecho paralelismo con lo que podríamos denominar el fracaso del programa cartesiano. El programa cartesiano fracasó al querer mostrar la autonomía plena de la razón y tuvo que reconocer en la intuición sensorial otra fuente del conocimiento objetivo de los objetos externos. De la misma manera, el programa de Hilbert, como se deduce de los teoremas de incompletitud de Gödel, fracasó al querer mostrar la plena autonomía de la razón formalizada. En ese sentido, el único recurso que le queda al matemático para salvar el carácter descriptivo de sus proposiciones, a juicio de Gödel, es apoyarse en la intuición de los conceptos abstractos. El argumento de Gödel exige que la mente humana no se pueda reducir a un mecanismo. Esta segunda exigencia es también paralela a la defensa del dualismo cartesiano.
\end{abstract}

Palabras clave: intuición, incompletitud, platonismo, dualismo

Nada se edifica sobre la piedra, todo sobre la arena, pero nuestro deber es edificar como si fuera piedra la arena.

JORGE LUIS BORGES ${ }^{\dagger}$

Gödel es considerado como uno de los matemáticos más importantes del siglo XX. En eso no hay ninguna duda. En los últimos veinte años ha surgido un especial interés por sus ideas filosóficas. Este interés ha sido motivado particularmente por la transcripción de las conversaciones sostenidas por el matemático con su amigo Hao Wang. En el caso de las comunidades académicas de habla hispana ha sido especialmente relevante la publicación de algunos escritos inéditos de Gödel a cargo del profesor Rodríguez Consuegra. ${ }^{1}$ El profesor Rodríguez sostiene, de una manera algo arriesgada,

* Trabajo presentado como requisito para ingresar a la Sociedad Colombiana de Filosofía en noviembre de 2001. Agradezco los juiciosos comentarios que recibí de parte del profesor Fernando Zalamea.

$\dagger$ Jorge Luis Borges, Fragmentos de un evangelio apócrifo, Obra poética, Emecé, Buenos Aires, 1992, § 41.

${ }^{1}$ Véase Rodríguez Consuegra 1994. El libro presenta la conferencia Gibbs dictada por Gödel en la reunión anual de la American Mathematical Society, en Brown University en 
que las principales motivaciones de Gödel eran precisamente filosóficas, que el trabajo que culminó en los teoremas asociados con la incompletitud tenían como motivación central la defensa de alguna forma de platonismo. $^{2}$ Creo que no es necesario, a la hora de esclarecer las ideas filosóficas del lógico matemático, adoptar, a partir de un soporte tan débil, una tesis tan extrema. Por otra parte, las ideas filosóficas de Gödel, que han llegado a nosotros por intermedio de Hao Wang, suelen estar impregnadas de las intenciones filosóficas de su interlocutor. No es fácil discernir en tales escritos el peso relativo de las ideas originales de Gödel del peso que adquieren las sugerencias de Hao Wang. De cualquier manera, tales conversaciones aportan testimonios importantes que deben ser evaluados con las reservas del

diciembre de 1951. También se incluyen las versiones II y VI del artículo que Gödel estaba preparando en homenaje a Carnap bajo el título: “¿Es la matemática sintaxis del lenguaje?” Este artículo finalmente no se publicó en la célebre serie "The Library of Living Philosophers" que Paul Schlipp editó en honor a la obra de Carnap. Al parecer, el artículo nunca alcanzó una versión que dejase plenamente satisfecho a Gödel. El libro cuenta también con una reseña e introducción del profesor Rodríguez Consuegra. En esta reseña se defiende una tesis extrema para explicar la producción de Gödel. Para apreciar la magnitud extrema de la tesis del profesor Rodríguez, véase, por ejemplo, la siguiente cita: "El realismo matemático será sucesivamente considerado como: (i) una consecuencia filosófica de tales resultados [se refiere a los teoremas de completitud e incompletitud]; (ii) un principio heurístico que conduce a ellos; (iii) una hipótesis filosófica que resulta verificada por ellos" (p. 23). A lo largo del presente artículo se aportan elementos para mostrar que (i) no es necesariamente correcta: el argumento es incompleto; (ii) es históricamente frágil (no contamos con los suficientes elementos para sostener tal afirmación, aun cuando reconocemos que algunos planteamientos filosóficos han podido estar presentes desde un momento temprano: algunas cartas a Hao Wang y algunos comentarios en sus escritos inéditos sugieren que el realismo platónico ya estaba presente en las inclinaciones de Gödel desde 1929; lo que no es del todo claro es que tales inclinaciones constituyeran la motivación principal e hicieran las veces de principios heurísticos para sus investigaciones lógicas). Por último, (iii) no se sigue directamente de tales resultados.

2 Véanse las anotaciones críticas a la tesis del profesor Rodríguez Consuegra sugeridas por el profesor Fernando Zalamea en "Kurt Gödel: análisis filosófico y lógica matemática". El profesor Zalamea se encuentra más conforme con una tesis intermedia a partir de la cual el análisis filosófico y la lógica matemática se encuentran en el mismo nivel en la producción del lógico austriaco. El lector puede encontrar también una tesis más prudente en el artículo de Feferman: "Kurt Gödel: Conviction and Caution". A juzgar por los artículos publicados por Gödel, es posible dividir su producción en dos periodos: antes de 1940 se publicaron sus principales contribuciones a la lógica matemática. Estos artículos se podrían calificar de filosóficamente asépticos. Gödel se encargó de depurar sus artículos de tal manera que no pudiera entreverse ningún compromiso filosófico (el profesor Zalamea no comparte esta perspectiva; véase Zalamea 1996, p. 358). Después de 1940, Gödel empezó a presentar, en forma tímida, algunas recomendaciones asociadas con la filosofía de las matemáticas. El artículo de 1944, "Russell's Mathematical Logic", marca, quizá, la diferencia entre los dos periodos. Gödel se sentía, como mostraremos en el presente artículo, por fuera del espíritu de la época. Es posible que este hecho determinara una actitud filosóficamente tímida y prudente en relación con sus primeros escritos. Tan pronto como Gödel pudo abandonar Viena, asentarse en Princeton y apreciar que sus contribuciones fundamentales eran generalmente reconocidas, como sugiere Feferman, se sintió más libre de expresar sus propias convicciones. La muerte de Hilbert ocurrió, casualmente, un año antes de la publicación del artículo de Gödel sobre Russell. Véase Feferman 1988. 
caso. En lo sucesivo me ocuparé de mostrar los argumentos que permiten reconstruir la defensa de alguna forma de platonismo a partir de algunas de las ideas que se derivan directamente de los escritos de Gödel. También pretendo mostrar que la defensa no alcanza cabalmente el propósito inicial.

Con el objeto de ubicar el contexto de la discusión deseo presentar inicialmente la taxonomía del problema que se deriva de la contribución de Mark Balaguer en su libro: Platonism and Anti-Platonism. ${ }^{3}$ El platonismo matemático puede ser presentado en varias versiones, de las cuales por el momento me interesa resaltar dos. La primera es una versión débil que puede formularse en los siguientes términos: ${ }^{4}$

(a) Existen objetos matemáticos tales como los números. Estos objetos no son espacio-temporales y existen independientemente de nosotros y de nuestra actividad teorizante.

(b) Nuestras teorías describen tales objetos.

La premisa (b) es fundamental, pues si afirmamos que hay objetos matemáticos pero que desafortunadamente no hay forma de conocerlos o de advertir su presencia, estaremos defendiendo una tesis inocua. Algo muy parecido a lo que ocurría con el clérigo que quería oponerse al descubrimiento de montañas en la Luna anunciado por Galileo. Este personaje sostenía que aunque pudiésemos ver montañas en la Luna, ella era en verdad esférica, pues estaba cubierta por una cúpula de cristal perfectamente esférica que por desgracia no podíamos contemplar haciendo uso del telescopio de Galileo ni de ningún instrumento óptico. ${ }^{5}$

La versión fuerte del platonismo se puede formular en estos términos: "Todo objeto matemáticamente posible existe." 6 De las dos versiones me interesa particularmente restringir el estudio al ámbito de la versión débil.

${ }^{3}$ Balaguer 1998.

${ }^{4}$ Esta presentación es una buena paráfrasis de la definición de platonismo que ofrece Gödel al final de la conferencia acerca de las implicaciones de algunos resultados matemáticos: "[Por una visión platónica de las matemáticas] quiero dar a entender que las matemáticas describen una realidad no sensible, la cual existe independientemente tanto de los hechos como de las disposiciones de la mente humana y que es sólo percibida por ella, aunque esto pueda ocurrir de forma incompleta" (SBT, p. 323).

${ }^{5}$ Conviene, por el fino humor que encierra, recordar la respuesta de Galileo: Aceptaremos la esfera de cristal que envuelve la Luna siempre que aceptemos también que tal esfera contiene protuberancias de cristal que tampoco podemos observar con el telescopio. Véase Galilei 1984, p. 198.

${ }^{6}$ Balaguer propone la siguiente paráfrasis no del todo adecuada: $\forall x[(x$ es un objeto matemático \& $x$ es lógicamente posible) $\rightarrow x$ existe]. La paráfrasis no es del todo adecuada y resulta problemática pues hace de la existencia un predicado e introduce elementos modales en una forma no muy clara. De cualquier manera, lo que nos interesa es resaltar, de modo pasajero, una sugerencia interesante en la forma de presentar una modalidad más fuerte de platonismo. 
Es decir, un platonismo comprometido con la existencia objetiva de los objetos matemáticos por fuera del espacio-tiempo, y no un platonismo que incorpore conceptos aún más complejos como el concepto de posibilidad lógica. ${ }^{7}$

Esta forma de plantear el platonismo da origen a dos tipos de respuestas antiplatónicas dependiendo de la actitud que se adopte frente a (a) o a (b). Por un lado, los antiplatónicos realistas, quienes defienden parcialmente (a), sostienen que, si bien las matemáticas describen alguna clase particular de objetos, tales objetos no son entidades abstractas no espaciotemporales. Los antiplatónicos realistas pueden defender que, o bien la matemática trata de objetos físicos (como lo hace el empirismo de J.S. Mill) y, en consecuencia, los matemáticos son descubridores; o bien la matemática trata de objetos mentales (como afirman Erdmann y Husserl) y los matemáticos son inventores. Por otro lado, los antiplatónicos antirrealistas, quienes se oponen completamente a (a) y a (b), sostienen que las matemáticas no describen ninguna clase de objetos. Es decir, las expresiones de la matemática carecen de contenido $\mathrm{y}$, en consecuencia, no describen un particular estado de cosas en algún mundo posible. Conviene citar en este caso tres variantes de antiplatonismo antirrealista: (i) el convencionalismo sostiene que las proposiciones de la matemática son analíticamente verdaderas: son verdaderas en virtud del significado de sus términos; (ii) el deductivismo afirma que las expresiones de la matemática son expresiones de la forma: "es necesario que si $A$, entonces T"; (iii) el formalismo sostiene que las matemáticas ofrecen verdades que se sostienen en el marco de ciertos sistemas formales: la matemática se ocupa de la manipulación de ciertos signos sujetos a ciertas reglas formales de transformación. Estas versiones de antiplatonismo tienden a identificar verdad y demostrabilidad.

Una de las críticas más fuertes contra el platonismo se puede sintetizar en el argumento presentado por Benacerraf en su artículo ya clásico "Mathematical Truth". 8 A juicio de Paul Benacerraf, la filosofía de las matemáticas, es decir, la disciplina que pretende aportar un esquema racional

\footnotetext{
${ }^{7}$ Un rasgo que ilustra la importancia de la posibilidad lógica como criterio de existencia puede subrayarse en el siguiente fragmento de una carta de Hilbert a Frege. No obstante, no pretendo sugerir que Hilbert haya defendido alguna clase particular de platonismo. He aquí el fragmento: "Si los axiomas arbitrariamente dados no se contradicen el uno al otro, entonces ellos son verdaderos y las cosas definidas por los axiomas existen. Éste es para mí el criterio de verdad y existencia" (Frege 1980, pp. 39-40). Hilbert pretende así responder a la tesis opuesta de Frege según la cual de la verdad de los axiomas se sigue que ellos no se contradicen. Aunque el resultado de Gödel asociado con la completitud del cálculo de predicados de primer orden parece justificar la relación entre existencia y consistencia, todos los trabajos posteriores pretenden desvirtuar la extensión generalizada de tal afirmación: el criterio de existencia de un objeto matemático no se puede reducir a la consistencia.

${ }^{8}$ En Benacerraf y Putnam 1983, pp. 403-420.
} 
para explicar la naturaleza tanto de las proposiciones matemáticas como de la actividad de los matemáticos, debe atender en forma articulada dos demandas complejas. Por un lado, cualquier teoría de la verdad matemática debe estar en conformidad con una teoría general de la verdad. En otras palabras, las adscripciones semánticas que asignemos a las proposiciones matemáticas deben ajustarse al mismo modelo con el cual hacemos adscripciones semánticas a otras proposiciones. Por otro lado, cualquier programa de investigación en filosofía de las matemáticas debe dar cuenta de la manera como obtenemos conocimiento matemático. En otras palabras, las condiciones de verdad de las proposiciones matemáticas no pueden hacer imposible para nosotros reconocer que ellas son satisfechas. En términos aún más claros, el concepto de verdad matemática debe encajar dentro de una explicación general del conocimiento en una forma tal que resulte inteligible cómo adquirimos nosotros el conocimiento matemático que poseemos. Las dos demandas se pueden resumir en los siguientes términos: una semántica adecuada para las matemáticas debe encajar en una epistemología aceptable. En ese orden de ideas, es posible advertir de antemano las dificultades del realismo platónico: si los términos matemáticos refieren a objetos no espacio-temporales, ¿cómo explicamos entonces el hecho de tener conocimiento de tales objetos con la epistemología aceptable?

Ahora bien, cuando Benacerraf habla de una epistemología aceptable tiene en mente una teoría causal del conocimiento. De hecho, Benacerraf se compromete también con una teoría causal de la referencia. ${ }^{9}$ En gracia al desarrollo de la discusión, obviaremos por lo pronto las dificultades que se derivan de tan problemático compromiso. El núcleo central del argumento antiplatónico se puede presentar en los siguientes términos: los pretendidos objetos matemáticos abstractos no espacio-temporales son causalmente inertes. Recojamos la estructura del argumento de Benacerraf valiéndonos de la síntesis de Balaguer:

(1) los seres humanos existen enteramente en forma espacio-temporal;

(2) si hay objetos matemáticos abstractos, ellos existen por fuera del espacio-tiempo;

(3) aplicando la teoría causal del conocimiento: si existen objetos matemáticos abstractos, los seres humanos no podrían tener conocimiento de ellos;

\footnotetext{
9 "Apoyo una teoría causal del conocimiento a partir de la cual para que $X$ sepa que $S$ es verdadera se requiere que exista alguna relación causal entre $X$ y los referentes de los nombres, predicados y cuantificadores de $S$. Además, creo en una teoría causal de la referencia, esto me obliga a admitir el conocimiento de $S$ como algo doblemente causal" (Paul Benacerraf 1973, p. 412).
} 
(4) si el platonismo matemático es correcto, los seres humanos no podrían tener conocimientos matemáticos;

(5) los seres humanos tienen conocimientos matemáticos;

(6) luego: el platonismo matemático no es correcto.

Las respuestas de los platónicos se pueden clasificar en tres alternativas dependiendo de la actitud que se adopte frente a (1), (2) o (3). En primer lugar, es posible negar (1) y afirmar que los seres humanos no son enteramente espacio-temporales y que es posible hablar acerca del contacto con otros mundos. En particular, ésta parece ser la estrategia que se ajusta en forma más adecuada al pensamiento de Gödel. En segundo lugar, es posible negar (2) y afirmar que los seres humanos pueden adquirir información acerca de los objetos matemáticos por medios perceptuales. Esta alternativa es defendida por Maddy. ${ }^{10}$ Por último, es posible negar (3) y apartarse de la teoría causal del conocimiento. Algunos defensores de tal alternativa son: Quine, Steiner, Parsons, Hale, Wright, Resnik. No obstante, conviene aclarar que un autor como Quine se opone a (3), no con el objeto de defender el platonismo, sino con el objeto de debilitar un argumento al estilo de Benacerraf. Quine defiende una forma de holismo del conocimiento por oposición a un esquema estrictamente causal. En lo sucesivo nos ocuparemos de la manera como se puede estructurar una defensa de la primera alternativa a partir de las ideas que se sugieren en los escritos originales de Kurt Gödel. No pretendo con ello sostener que se trata de la mejor defensa; de hecho, la tercera alternativa constituye, a mi juicio, la mejor defensa en caso de que hubiese todavía algún recurso para el platonismo.

No son muchos los artículos de Gödel a partir de los cuales es posible construir la visión canónica del autor frente a los problemas filosóficos. Algunos de ellos permanecieron inéditos hasta hace poco tiempo. Ello debido posiblemente a algunos rasgos peculiares del carácter de Gödel: él era especialmente celoso con escritos que aún no habían alcanzado las exigencias de calidad que el mismo autor se había impuesto. Por otra parte, creo yo, sus ideas filosóficas no lograron abandonar el estado de reflexiones heurísticas. No era fácil encontrar un interlocutor para las ideas filosóficas de Gödel, pues, de alguna manera, estaban aún sumergidas en la niebla metafísica de los siglos XVII y XVIII. El interlocutor ideal, sin duda, habría sido Leibniz. De cualquier manera, es posible resaltar algunos rasgos esenciales y algunas ideas brillantes que hacen del pensamiento de Gödel un pensamiento digno de exploración y consideración. Me ocuparé inicialmente de la actitud filosófica que Gödel, en forma panorámica, tenía hacia los programas de fundamentación de la matemática. Para ello, quiero hacer

10 Véase Maddy 1990. 
uso del artículo titulado "The Modern Development of the Foundations of Mathematics in the Light of Philosophy", publicado en el tercer volumen de los Collected Works. Gödel se hizo miembro de la American Philosophical Society en 1961 y es posible que hubiese escrito tal artículo con el objeto de cumplir con el acostumbrado requisito de ofrecer una corta charla a los miembros de la sociedad.

Gödel comienza por dividir las visiones-del-mundo [Weltanschauungen] de acuerdo con el grado de afinidad o distancia con respecto a la metafísica o a la religión. Este ejercicio lo lleva a proponer la siguiente disposición en dos grupos: escepticismo, materialismo y positivismo de un lado, y espiritualismo, idealismo y teología del otro. El esquema le permite hablar de las visiones de izquierda y de derecha respectivamente. No hay claridad acerca de las distinciones que desea trazar, pero las intenciones se dejan ver con absoluta nitidez. A priorismo y optimismo son rasgos de las visiones de derecha, en tanto que empirismo y pesimismo lo son de las visiones de izquierda. A manera de ejemplo: "El materialismo está inclinado a contemplar el mundo como una desordenada aglomeración de átomos y, en consecuencia, carente de significado" (DFM, p. 375). Esto nos conduce a contemplar la muerte como una completa aniquilación final. En contraste, la teología y el idealismo permiten detectar sentido, propósito y razón en todo. ${ }^{11}$

El siguiente paso en la argumentación consiste en proponer, con cierto tono de lamento, la siguiente descripción del desarrollo de la filosofía desde el Renacimiento hasta nuestros días: la filosofía se ha desplazado como un todo desde la derecha hacia la izquierda, no siempre en línea recta continua, sino practicando ciertos tímidos retrocesos. Presentemos en un esquema el panorama que ilustra Gödel:

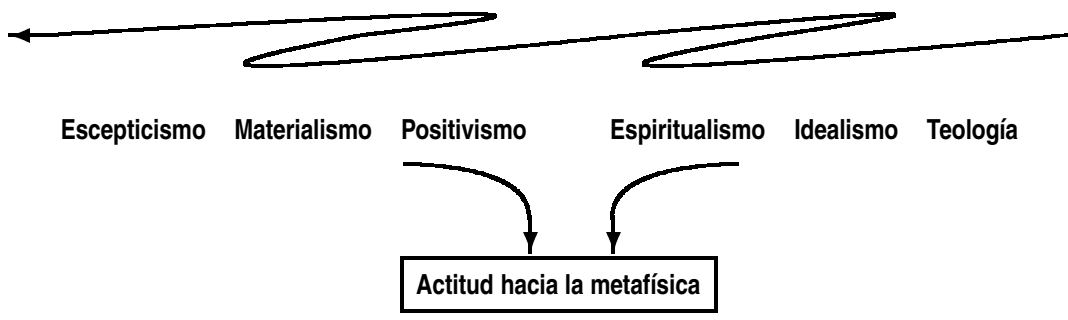

Este movimiento se advierte claramente en el desarrollo alcanzado por las ciencias físicas durante el cambio de siglo: nos desplazamos desde una confianza plena en los alcances del programa mecanicista hacia la fragilidad

\footnotetext{
${ }^{11}$ Hao Wang ha sugerido que la división de Gödel en visiones del mundo a la izquierda y visiones a la derecha es una reminiscencia de un contraste introducido por William James. Véase Wang 1996, p. 162.
} 
de un conocimiento que debe contentarse con predecir resultados de observaciones, renunciando así a los deseos de explicación. Las matemáticas, a la luz del lógico más grande del siglo xx, se han caracterizado siempre por una tendencia inamovible hacia la derecha. Gracias a ello, y por tratarse de una ciencia a priori, supo mantenerse ajena al espíritu de la época. No hizo mella, por ejemplo, la escandalosa intervención del empirismo de J.S. Mill. No obstante, tarde o temprano tendría que caer en manos de la seducción izquierdista. Es muy posible que Gödel leyera el siguiente hermoso fragmento de Frege como la conspiración de un anarquista: después de mostrar que en el caso de las matemáticas no basta un convencimiento puramente moral apoyado en muchas aplicaciones convincentes, y "Después de que uno se haya convencido de la imposibilidad de mover una roca ante la inutilidad de los esfuerzos por lograrlo, se puede preguntar qué

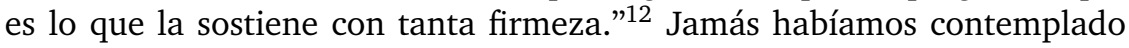
tal irreverencia: atreverse siquiera a cuestionar los fundamentos mismos de la matemática. El mismo Descartes tuvo que inventarse un argumento esotérico para poner en entredicho, en forma pasajera, el piso firme de las matemáticas: imaginar un genio maligno que me hace concebir en forma clara y distinta aquello que no es verdadero. Pero este atrevimiento de Frege roza ya los límites de la insolencia: convertir la petición pasajera, y en algunos aspectos inocua, de Descartes en un programa permanente de investigación. La falibilidad, propia de las ciencias empíricas, pronto se transformó en una clara amenaza para las matemáticas. Gracias a la proliferación de nuevas geometrías y en virtud del escándalo generado por las antinomias que surgieron en la teoría de conjuntos y de la exigencia de un programa de investigación que estipulara los fundamentos de la matemática, la falibilidad adquirió la forma del imperativo de una prueba de consistencia para los sistemas matemáticos.

El programa de Hilbert, en términos de la valoración de Gödel, posee una curiosa propiedad hermafrodita: pretende hacer justicia tanto al espíritu de la época (inclinación hacia la izquierda) como a la naturaleza de la matemática (inclinación hacia la derecha). No sé si exista un término adecuado para este tipo de personaje en ámbitos políticos. Por un lado, ajustado al espíritu de la época, reconoce que la verdad de los axiomas a partir de los cuales se edifica la matemática no puede ser justificada plenamente $\mathrm{y}$, en consecuencia, los resultados de la matemática tienen únicamente significado en un sentido hipotético. Tales consecuencias son entonces el resultado de un mero juego con símbolos inertes. Por el otro, y con el objeto de aludir a la naturaleza de la matemática, el programa de Hilbert sugiere que cada cuestión matemática debe poderse formular de manera

12 Frege 1950, § 2, p. 2. 
precisa como una pregunta que demanda una y sólo una de dos respuestas posible: sí o no, y, además, debe poderse responder en forma definitiva.

La idea central de Gödel ante el programa de Hilbert, tal y como él lo concibe, puede sintetizarse en los siguientes términos: no es posible rescatar los viejos aspectos hacia la derecha de los programas de investigación en matemáticas de una manera tal que se encuentren más o menos en armonía con el espíritu de la época que impone una clara tendencia hacia la izquierda. El argumento fuerte para defender tal perspectiva se deriva directamente de una pretendida consecuencia filosófica de los teoremas de incompletitud. ${ }^{13}$ En primer lugar, si nos limitamos a la teoría de los números naturales, ${ }^{14}$ es imposible encontrar un sistema de axiomas y reglas formales a partir de los cuales, para cada proposición teórica de números $A$, o bien $A$ o $\neg A$ sea derivable. En otras palabras, no existe un sistema formal consistente en el cual sean derivables todas las traducciones de las proposiciones que se tienen por verdaderas en la teoría de números. En segundo lugar, es imposible ofrecer una prueba que muestre la consistencia del sistema valiéndose tan sólo de combinaciones concretas de símbolos sin introducir más elementos abstractos. De lo anterior se derivan, pues, dos alternativas antagónicas: renunciar a los aspectos de derecha provenientes de la naturaleza de la matemática, o defenderlos en contradicción con el espíritu de la época. Gödel, como Leonardo Da Vinci, se sentía fuera de la época en la que se vio obligado a vivir. No precisamente porque Gödel y Leonardo hubiesen avizorado tiempos por venir, sino porque se habrían sentido más a gusto con interlocutores que se quedaron atrapados en el pasado. Leonardo habría disfrutado sesiones inolvidables al lado de Platón, contra la opinión corriente que esperaría que el viejo constructor disfrutase más aquella época que materializó sus proyectos de ingeniería. Gödel habría disfrutado sesiones inolvidables al lado de Leibniz, contra la opinión corriente que esperaría que el viejo lógico disfrutase la época que materializó las máquinas de Turing.

En este orden de ideas, Gödel concluye:

13 Hoy en día ya no es tan claro que los teoremas de incompletitud hayan liquidado en forma definitiva las pretensiones del programa de Hilbert. En ese orden de ideas, resultan especialmente importantes las observaciones de Detlefsen. Véase Detlefsen 1990. El profesor Zalamea sugiere también una relativización, más que una aniquilación, del programa: "Corresponde mejor al desarrollo de aspectos de la lógica matemática a partir de Gödel [.. .] afirmar que el programa de Hilbert - que pretendía alcanzar un ideal absoluto de consistencia- no murió para siempre, sino que más bien se relativizó en diversas maneras muy profundas, con lo que en vez de derrumbarse ha alcanzado una influencia tal vez mayor" (Zalamea 1996, p. 355). Véanse también los programas de investigación en matemáticas en reversa. No obstante lo anterior, nos interesa, más bien, considerar el tipo de evaluación que practicaba Gödel.

${ }^{14}$ Gödel está pensando en la aritmética de Peano. 
Uno debe decir que la fecundidad del materialismo está en parte basada únicamente en la inmoderación y la dirección equivocada de la filosofía de derecha que le precedió. Tan pronto como nos ocupemos de la corrección o inexactitud, o, respectivamente, verdad y falsedad, de estas dos direcciones, la actitud correcta nos mostrará que la verdad yace en el medio o que consiste en una combinación de las dos concepciones. (DFM, p. 381)

Gödel quiso mostrar que el intento de Hilbert era un proyecto de antemano condenado al fracaso porque se inclinaba fuertemente hacia una de las dos direcciones. Creo que el espectro de posibilidades que explora Gödel es, sin duda, muy limitado. Es posible pensar en un esquema en el cual todas las alternativas que ofrece Gödel correspondan, por igual, a un problema mal planteado. Ninguno de ellos constituye, en estricto sentido, un programa de investigación legítimo. Ésta es, por ejemplo, la perspectiva que se deriva del análisis terapéutico de Wittgenstein. Gödel, en particular, se sentiría especialmente distante de este tipo de reflexión por sugerir una especial atención a la gramática de nuestro lenguaje. Él siempre tuvo una actitud displicente hacia los planteamientos que hacían del lenguaje el centro de la reflexión. ${ }^{15}$ De cualquier manera, no nos interesa por lo pronto dar con la alternativa más acertada, sino tratar de organizar las perspectivas filosóficas de Gödel. Obviaremos, entonces, la alusión a otros esquemas o posiciones.

La estrategia argumentativa de Gödel guarda también cierto parentesco con la estrategia de Descartes hacia el escepticismo. Descartes veía en el escepticismo un serio peligro tanto para el fundamento de las creencias religiosas como para el fundamento último de la ciencia. El programa cartesiano puede verse, de alguna manera, como reacción al espíritu de la época. Los detractores del escepticismo se habían visto envueltos en estrategias estériles que no lograban responder con vehemencia a los argumentos que pretendían eliminar. La actitud del escéptico tenía siempre la ventaja de la prudencia, la ventaja de aquel que se compromete con menos. El dogmático, por el contrario, tenía sobre sus hombros la carga de la prueba. Es él quien debe probar que el mundo existe, es él quien debe mostrarnos que hay un fundamento sólido para el conocimiento. El escéptico puede limitarse a contemplar con cierto aire de incertidumbre. ¿Cuál fue entonces la estrategia de Descartes? Sumergirse en el escepticismo hasta las raíces más profundas, llevar los propios argumentos escépticos hasta los límites más extremos para demostrar que aún así es posible salir del atolladero, que es posible edificar una construcción sólida sembrando ladrillo sobre ladrillo. En otras palabras: adoptar la estrategia metodológica del escepticismo para fracturarlo desde el interior. Algo parecido ocurre con Gödel, siempre que estemos dispuestos a contemplar el programa de Hilbert como una

$15 \mathrm{Al}$ respecto, véanse algunas anotaciones en Wang 1996. 
instauración de las pretensiones escépticas en el ámbito de la investigación matemática. No creo que esta interpretación logre capturar en esencia los alcances del programa de Hilbert, pero creo, al menos, que sí logra dar cuenta de la actitud de Gödel hacia el programa. Gödel aceptó la estrategia metodológica del programa de Hilbert —al menos en las pretensiones formalistas y logicistas - para fracturarlo desde el interior. ${ }^{16}$ En los reportes que Gödel aportó a Hao Wang acerca de las estrategias que lo llevaron a los teoremas de incompletitud se sugiere el siguiente procedimiento: Gödel empezó por estudiar el problema de Hilbert de probar la consistencia del análisis por medios finitistas; encontró después algo misteriosa esta restricción sobre los métodos de prueba e intentó probar la consistencia de la teoría de números por la teoría de números finitista y la consistencia

${ }^{16}$ La comparación entre la estrategia de Descartes y la de Gödel no puede exponerse con éxito en todos sus detalles. Creo que hay puntos en los cuales la comparación no favorece a Gödel. Es posible sugerir, por ejemplo, que el programa cartesiano no pudo formularse con el éxito que terminamos reconociéndole. Por un lado, la demostración de la existencia de Dios es absolutamente cuestionable, y, por el otro, la supuesta demostración bien sea de la existencia del mundo exterior o de la existencia de otras mentes adolece de dificultades insalvables. En ese orden de ideas, bien podríamos pensar que Descartes, a pesar de sus buenas (!) intenciones, terminó haciéndole un favor espectacular al escepticismo. No obstante lo anterior, podríamos sugerir otro tipo de lectura para salvar la comparación. Podríamos pensar que la reconstrucción del mundo que pretende adelantar Descartes a partir de elementos que no reconocen ninguna clase de compromiso, guarda cierto parecido con la reconstrucción de las verdades matemáticas que pretende adelantar Hilbert a partir de elementos que no reconocen ninguna clase de compromiso. En ese orden de ideas, el fracaso del programa cartesiano - es decir, la imposibilidad de la razón para establecer por sus propios medios la existencia del mundo exterior y verse, por esa razón, en la obligación de acudir a un recurso diferente: la intuición sensorial- guarda un estrecho parecido con el supuesto fracaso del programa de Hilbert. Gödel, creía él, puso en evidencia la incapacidad tanto del formalismo como del logicismo para establecer por sus propios medios una y cada una de las verdades de la teoría de números. Gödel mostró que en todo sistema formal que pretenda capturar todas las verdades de la teoría de números es posible advertir al menos una verdad que no puede ser capturada. También puso en evidencia, a su juicio, la necesidad de acudir a un recurso diferente: la intuición matemática. Es importante tener en mente el esquema de argumentación de Descartes para efectos de resaltar los rasgos comparativos que nos interesan. Después de poner en duda todas las proposiciones que se tenían por verdaderas, después de establecer el carácter inamovible de la proposición: "pienso, luego soy" y después de demostrar la existencia de Dios y establecer que todo aquello que captamos en forma clara y distinta es verdadero, Descartes procede, al final de la quinta meditación y durante la sexta, a probar que el mundo exterior existe. En la quinta meditación prueba la realidad de la esencia del mundo: en caso de existir, la esencia del mundo radica en la extensión. En la sexta meditación se apoya en la realidad de la esencia para mostrar la posibilidad de la existencia del mundo exterior. El argumento central consiste en mostrar que no hay contradicción alguna. Apoyado después en la imaginación, concluye que es altamente probable que el mundo exterior exista. Por último, es necesario apoyarse en la sensibilidad para concluir con certeza la existencia del mundo exterior. Descartes propone la existencia de una facultad pasiva encargada de albergar las imágenes provocadas por cierta facultad activa que debe originarse en una fuente independiente del espíritu. Es precisamente este último punto el que muestra el carácter incompleto de la razón para adelantar la tarea que ella misma se había impuesto. La intuición sensible llena el vacío que ha dejado abierto la razón. 
del análisis por la teoría de números. Gödel, según los relatos de Wang, representó los números reales mediante fórmulas de la teoría de números y encontró que tenía que usar el concepto de verdad para las proposiciones en la teoría de números con el ánimo de verificar algunos axiomas del análisis. Esto lo condujo a algunas paradojas relacionadas con la verdad y su definición. Si la verdad para la teoría de números fuera definible en el interior de ella misma, podría encontrarse una versión precisa de la sentencia del mentiroso y, en consecuencia, llegar a una contradicción. No obstante, la noción de derivabilidad sí es definible; por lo tanto, derivabilidad y verdad deben ser conceptos independientes. Gödel notó que la verdad en la teoría de números no puede ser definida en tal teoría y, en consecuencia, su plan inicial fracasó, y con ello el programa de Hilbert. ${ }^{17}$ En una breve reseña de un artículo de Carnap, Gödel reconoce que Carnap había advertido con claridad que una noción como la de verdad no puede ser expresada en un lenguaje consistente. ${ }^{18}$

Gödel concluyó, entonces, que de los teoremas de incompletitud se deriva que la certeza de las matemáticas no se asegura después de probar ciertas propiedades por una proyección sobre sistemas de símbolos ajustados a ciertas reglas de transformación. La certeza en las matemáticas debe tener otra fuente: la intuición matemática. No podemos eludir el recurso al conocimiento de los conceptos abstractos. ¿Cómo es, entonces, posible extender nuestros conocimientos hacia los dominios de los conceptos abstractos? Ello no se logra aportando definiciones explícitas para conceptos y pruebas a partir de axiomas. Esto nos encerraría en un círculo vicioso. "El procedimiento", recomienda Gödel, "debe consistir, al menos en una gran extensión, en una clarificación del significado que no consiste en dar definiciones" (DFM, p. 383). Si atendemos la recomendación haciendo abstracción del contexto, es posible advertir una clara semejanza con los proyectos de análisis conceptual wittgensteiniano. La diferencia central reside en el

17 Véase Wang 1981, "Some Facts about K. Gödel”, pp. 653-659; específicamente la p. 654. En una carta de Gödel a Hao Wang se aclara el principio heurístico del lógico matemático en los siguientes términos: "debe notarse que el principio heurístico de mi construcción de proposiciones indecidibles de la teoría de números en los sistemas formales de la matemática es la oposición entre el concepto de 'verdad matemática objetiva' y el concepto de 'demostrabilidad'" (citada en Wang 1974, p. 9). Aunque el realismo platónico es importante en las ideas de Gödel, el principio heurístico de su investigación es la oposición entre los conceptos de verdad y demostrabilidad. También es cierto que a partir de dicha oposición se pretende argüir una defensa del platonismo. Lo que no se desprende de la carta es que el platonismo sea el principio heurístico de la investigación, como pretende el profesor Rodríguez Consuegra (véase la nota 1 del presente artículo). Aun el convencionalista podría orientar sus investigaciones imaginando la descripción de algunos objetos hipotéticos. Gödel formuló tal posibilidad en una nota a la conferencia Gibbs: "los nominalistas no negarían que nosotros realmente imaginamos objetos (no existentes) detrás de los símbolos matemáticos y que tales ideas subjetivas podrían aún aportar los principios guía en la elección de las reglas sintácticas" (SBT, p. 315, nota 23 ).

18 Véase CWI, p. 389. 
hecho de que la clarificación conceptual conduce a Wittgenstein a disolver la dificultad, en el sentido en el que se logra exhibir que la dificultad estaba mal planteada desde el comienzo; en tanto que la clarificación perseguida por Gödel debe conducir a la solución acertada de un problema bien planteado desde sus orígenes. Gödel promete llevarnos a la solución correcta; Wittgenstein promete liberarnos de una molestia inocua.

Con el ánimo de responder a la pregunta: “¿Cómo es posible tener conocimiento de los conceptos abstractos?", Gödel cree que es posible refugiarse en la fenomenología de Husserl. Sin embargo, las referencias a Husserl no son del todo claras y tampoco son juiciosamente explícitas. ${ }^{19}$ De cualquier manera, no aportan elementos sustanciales en la línea de argumentación que estoy desarrollando en favor de una forma débil de platonismo matemático. Por esa razón no hay inconveniente en que hagamos a un lado este sendero de exploración. Regresemos entonces al tema relacionado con el conocimiento de los conceptos abstractos. Creo que en este caso conviene resaltar dos puntos que convergen. Por un lado, la analogía que Gödel pretende defender entre una investigación física y una investigación matemática y, por otro lado, la nueva orientación que pretende Gödel de la concepción kantiana de las matemáticas. Ocupémonos, por ahora, del segundo aspecto. El conocimiento de los conceptos abstractos debe proceder por medio de una clase particular de intuición. El entendimiento intuitivo de nuevos axiomas, lógicamente independientes de un conjunto inicial de axiomas, por ejemplo, está de acuerdo en principio con la concepción kantiana de las matemáticas. Sin embargo, Kant sostiene que para efectos de la derivación de teoremas geométricos, necesitamos siempre nuevas intuiciones geométricas. Gödel sostiene que esta posición de Kant tomada literalmente es, sencillamente, falsa. No obstante, si reemplazamos el término "geométrica" por el término "matemática", entonces la posición de Kant resulta ser, a juicio de Gödel, verdadera. Hay, sin embargo, grandes diferencias entre la intuición kantiana y la intuición gödeliana tal y como lo ha puntualizado el profesor Hintikka: ${ }^{20}$

${ }^{19}$ Hao Wang encuentra igualmente incompleta e insatisfactoria esta referencia a Husserl: "Gödel parece sugerir (como un ideal) que deberíamos animarnos a usar el método fenomenológico para descubrir los axiomas por los conceptos primitivos de la filosofía. Pero no tengo conocimiento de que algunos conspicuos y exitosos ejemplos de axiomas definitivos hayan surgido de esa manera. Ni el axioma de elección, [...] ni siquiera los axiomas de Dedekind para la aritmética se obtuvieron al regresar a los actos últimos y a los contenidos de nuestra conciencia en la forma recomendada por la fenomenología" (Wang 1996, p. 159). Convendrá estudiar con atención si el recurso a la fenomenología de Husserl acerca a Gödel peligrosamente a alguna forma de psicologicismo. Véase, por ejemplo, la siguiente cita: "La fenomenología", advierte Gödel, "regresa a los fundamentos de nuestro conocimiento, al proceso de cómo nosotros formamos el conocimiento y descubre lo que nos es dado desde el interior" (citado en Wang 1996, p. 167).

${ }^{20}$ Gödel también era consciente de algunas diferencias: "Una buena traducción inglesa del término kantiano Anschauung es kantian intuition o concrete intuition. Las consideraciones 
La intuición kantiana puede implicar tal conocimiento [el conocimiento a priori] porque su mera introducción reproduce las operaciones a través de las cuales nosotros imponemos las formas de espacio y tiempo sobre los objetos y a través de las cuales hemos también individualizado aquellos objetos. En otras palabras, podemos anticipar intuitivamente la aplicabilidad de aquellas formas de experiencia porque nosotros mismos las hemos proyectado a los objetos [...]. De acuerdo con Kant, el uso de intuiciones a priori no es como percibir un objeto; es como introducir una representación para algún objeto particular desconocido en anticipación de cualquier conocimiento perceptual. Esto se encuentra en agudo contraste con Gödel, para quien la intuición podría acceder a una realidad independiente de la mente. ${ }^{21}$

En otras palabras, lo que Hintikka sugiere es que, aun cuando las categorías del entendimiento puro de Kant desempeñan un papel central en determinar lo que es objetivo en nuestras formas de representación, tales categorías no son objetivas en el sentido de representar un aspecto de las cosas o las cosas mismas.

No es nuestro interés juzgar si Gödel interpretaba en forma adecuada o no el pensamiento de Kant. De cualquier manera, sí conviene resaltar las prevenciones que Gödel, en forma justificada o no, tenía frente al autor de la Crítica de la razón pura. El artículo titulado "Some Observations about the Relationship between Theory of Relativity and Kantian Philosophy" (TRKP) deja ver algunas reacciones interesantes de Gödel frente a la filosofía crítica. En primer lugar, quiero resaltar que se trata de un documento excelente contra aquellas personas que en forma apresurada pretenden sostener que la teoría de la relatividad invalidó, en forma definitiva, las ideas de Kant; especialmente aquellos que sostienen que el empleo de las geometrías no euclidianas deja sin piso el pretendido carácter a priori de la geometría euclidiana en la obra de Kant. ${ }^{22}$ El artículo de Gödel comienza por advertir que él no se considera un partidario de la filosofía kantiana en general. Deseo resaltar especialmente un punto en relación con el tema que nos ocupa.

A mí me parece —afirma Gödel— que únicamente en un punto existe una contradicción real entre la teoría de la relatividad y la filosofía kantiana, a

kantianas de la intuición pura fallan en producir una bien fundamentada creencia en la consistencia de la aritmética. Ésta es una razón para rechazar a Kant. Nuestra intuición nos dice la verdad, no sólo de que 7 más 5 es 12, sino también de que hay infinita cantidad de números primos y que la aritmética es consistente" (citado en Wang 1996, p. 217).

21 Hintikka 1998, p. 21.

${ }^{22}$ Es muy posible que Gödel haya considerado, de alguna manera, el tema desarrollado en la tesis doctoral de Carnap, quien se ocupó de la tensión existente entre los fundamentos de la geometría y la nueva física. Carnap sugiere allí que se distinga entre espacio físico, intuitivo y formal; sugiere, además, que el espacio intuitivo es, efectivamente, euclidiano y a priori, como recomienda Kant. 
saber, la opinión de Kant según la cual la descripción del mundo dada por las ciencias naturales debe necesariamente detenerse ante las formas de nuestra percepción sensorial y no puede hacer nada más sino establecer relaciones entre apariencias en el interior de esta estructura. (TRKP, p. 244)

Esta dificultad proviene, a juicio de Gödel, de nuestra imposibilidad de conocer las cosas-en-sí-mismas. De ella también se deriva la necesidad de hacer transformaciones severas en la filosofía de Kant para ajustarla en forma más adecuada a las exigencias de la física contemporánea. Esta reforma, cree Gödel, permitiría a la filosofía crítica ir más allá de las apariencias y acercarse más al mundo de las cosas. Ahora bien, ¿por qué se molesta tanto Gödel con la distinción entre apariencias y cosasen-sí-mismas? Yo creo que esto se puede entender mejor si pensamos en las incomodidades que dicha tesis tendría a propósito de la naturaleza de las proposiciones matemáticas. La defensa de Gödel del realismo exige que exista una analogía estrecha entre una investigación física y una investigación matemática. Esto nos conduce al primer punto mencionado unas líneas antes. Los objetos de la física y los objetos de la matemática son necesarios para que tanto las proposiciones de la física como las proposiciones de la matemática tengan contenido. Los primeros nos son dados en la intuición sensible y los segundos en la intuición matemática. Si nuestra investigación matemática se detiene en el ámbito de la apariencia y no contamos con un acceso directo e inmediato a los conceptos abstractos, abrimos de par en par las puertas del escepticismo en matemáticas. En particular, no podríamos ya defender que la mente humana, como se pretende mostrar más adelante, supera las posibilidades de una máquina de Turing. Este punto debe hacerse más claro en las líneas que siguen.

El paralelo entre intuición matemática y percepción sensible va de la mano con el paralelo entre investigación matemática e investigación física. En el año de 1947 y a raíz de la hipótesis del continuo que surge en el tratamiento de los números transfinitos, Gödel había advertido que si se presupone la consistencia de los axiomas de la teoría de conjuntos, habría únicamente tres posibilidades para la conjetura de Cantor. Tal conjetura puede ser demostrable, refutable o indecidible. Gödel en ese entonces se inclinaba por esta tercera alternativa (CCP, p. 181). En 1963, después de una petición de Paul Benacerraf y Hilary Putnam, Gödel escribió un suplemento al artículo de 1947. En dicho suplemento, Gödel quiere responder a una de las dificultades que se derivarían en caso de que se pudiese demostrar la indecidibilidad de la conjetura de Cantor. Esta dificultad señala que en ese caso perdería significado la cuestión relacionada con la verdad de la conjetura, de la misma manera que la pregunta por la verdad del quinto postulado de la geometría euclidiana perdió su sentido una vez que 
fue demostrada la consistencia de algunas geometrías no euclidianas. Así como es posible hacer geometría euclidiana y no euclidiana, de la misma manera debe ser posible hacer teoría de conjuntos continuista y teoría de conjuntos no continuista. La respuesta de Gödel a la objeción no sólo fue enfática, sino claramente cargada del espíritu de derecha advertido unas páginas antes:

Una cuestión pierde su significado a causa de una prueba de indecidibilidad sólo cuando el sistema de axiomas en consideración se interpreta como un sistema formal; esto es, si se deja indeterminado el significado de los signos primitivos. En geometría, por ejemplo, la cuestión de si el quinto postulado de Euclides es verdadero conserva su significado si se toman los signos primitivos en un sentido definido, es decir, refiriéndose al comportamiento de cuerpos rígidos, rayos de luz, etc. La situación es parecida en la teoría de conjuntos y la única diferencia reside en que el significado que hoy usualmente se acepta en geometría se refiere a la física y no a la intuición matemática y, en consecuencia, la decisión cae fuera de las matemáticas. Por otro lado, los objetos de la teoría de conjuntos transfinita [...] no pertenecen al mundo físico e incluso su conexión indirecta con la experiencia es muy remota. (CCP, p. 267)

La importancia del problema de la verdad del quinto postulado está asociada con la necesidad de describir un marco de objetos cuya existencia y naturaleza es independiente de la geometría. De la misma forma, la importancia de la conjetura de Cantor se deriva de la necesidad de describir una realidad objetiva que en forma independiente sirva de soporte para la teoría de conjuntos. Nosotros tenemos acceso a esa realidad de una manera análoga a como accedemos a los objetos físicos. Gödel completa así su argumentación:

Pero, a pesar de su lejanía de la experiencia sensible, tenemos algo parecido a una percepción de los objetos de la teoría de conjuntos, como se puede ver por el hecho de que los axiomas mismos nos fuerzan a aceptarlos como verdaderos. No veo ninguna razón por la cual debamos tener menos confianza en ese tipo de percepción, es decir, en la intuición matemática, que en la percepción sensible, que nos induce a construir teorías físicas y a esperar que futuras percepciones sensibles concuerden con ellas y, además, a creer que cuestiones no decidibles por el momento tengan significado y puedan ser decididas en el futuro. (CCP, p. 268)

El paralelo entre la exigencia de objetos físicos y la exigencia de objetos matemáticos, para que tanto las proposiciones de la física como las proposiciones de la matemática tengan contenido, se aprecia con claridad en el artículo que Gödel escribió a propósito de la lógica matemática de Russell: 
Me parece que la suposición de tales objetos [Gödel se refiere a las clases y conceptos como objetos reales] es tan legítima como la suposición de los cuerpos físicos [...]. Ellos son en el mismo sentido necesarios para obtener un sistema satisfactorio de las matemáticas como los cuerpos físicos son necesarios para una teoría satisfactoria de nuestras percepciones sensoriales, y en ambos casos es imposible interpretar las proposiciones que uno quiere aseverar acerca de estas entidades como proposiciones acerca de los "datos". (RML, p. 128)

De no existir los objetos físicos, para nosotros sería absolutamente intrascendente hacer geometría euclidiana o geometría no euclidiana. La intuición que tenemos de la presencia de los objetos físicos nos obliga a inclinarnos hacia una clase particular de geometría. De la misma manera, la intuición de los objetos matemáticos debería inclinarnos a favorecer una teoría de conjuntos particular. No obstante, el argumento de Gödel parece circular: la intuición matemática favorece una clase particular de teoría de conjuntos y es precisamente el hecho de sentirnos inclinados hacia una clase de teoría de conjuntos lo que nos lleva a pensar en la existencia de una intuición matemática. El realismo de los objetos matemáticos desvanece la intrascendencia de los fenómenos de indecidibilidad. A su vez, este fenómeno de indecidibilidad, unido a un claro sentimiento de derecha, es el que nos lleva a postular la existencia de los objetos matemáticos. Estas reflexiones dejan ver que el parentesco con el destino del programa de Descartes es más estrecho que lo que habíamos pensado inicialmente.

La intuición matemática — concluye Gödel— no tiene que ser concebida como una facultad que proporcione un conocimiento inmediato de los objetos que le conciernen. Parece más bien que, como en el caso de la experiencia física, formamos también nuestros conceptos de estos objetos a partir de algo más que es inmediatamente dado. Sólo que este algo más no es aquí, o no principalmente, la sensación. ${ }^{23}$ Que además de las sensaciones hay algo real e inmediatamente dado se sigue (independientemente de las matemáticas) del hecho de que incluso nuestros conceptos referentes a los objetos físicos contienen constituyentes cualitativamente diferentes de las sensaciones o meras combinaciones de sensaciones; por ejemplo, el concepto mismo de objeto. Mientras que, por otro lado, mediante nuestro pensamiento no podemos crear ningún elemento cualitativamente nuevo, ${ }^{24}$ sino sólo reproducir y combinar los que están ya dados. Lo "dado" que subyace en las matemáticas está, evidentemente, muy relacionado con los elementos abstractos contenidos en nuestros conceptos empíricos. [Los datos de este segundo tipo] pueden representar un aspecto de

${ }^{23} \mathrm{Al}$ final de la quinta meditación, aún antes de que la intuición sensible nos muestre que hay objetos externos, Descartes muestra que es una condición impuesta por la razón el que, en caso de existir objetos externos, su esencia debe resumirse en la extensión.

${ }^{24}$ Insisto en que esta parte del argumento corre paralela al fracaso del programa cartesiano cuando pretende establecer, con el solo ejercicio de la razón, la existencia objetiva, no sólo posible, del mundo exterior. 
realidad objetiva, pero, en oposición a las sensaciones, su presencia en nosotros puede deberse a otro tipo de relación entre la realidad y nosotros mismos. (CCP, p. 268)

Para Gödel, la cuestión de la existencia objetiva del mundo exterior es una réplica de la cuestión de la existencia de los objetos matemáticos. Él se ve en la obligación de conjeturar una contrapartida de la percepción sensorial para el segundo mundo habitado por entidades abstractas. El problema sigue siendo confuso: ¿se deriva la existencia de los objetos matemáticos a partir de la intuición matemática que complementa las limitaciones detectadas en un sistema, de la misma manera como la existencia de los objetos físicos se deriva a partir de la intuición sensible que complementa las limitaciones detectadas en el ejercicio de la razón, tal como se concluye al valorar en forma panorámica el programa cartesiano? ¿O postulamos la necesidad del recurso de la intuición matemática con el objeto de salvar el problema del conocimiento de objetos abstractos? No creo que haya una respuesta satisfactoria a partir de los presupuestos de Gödel.

Recapitulemos. El argumento de Benacerraf contra el platonismo se apoya esencialmente en tres premisas: (1) los seres humanos existen enteramente en forma espacio-temporal; (2) si hay objetos matemáticos abstractos, ellos existen por fuera del espacio-tiempo; y (3) aplicando la teoría causal del conocimiento: si existen objetos matemáticos abstractos, los seres humanos no podrían tener conocimiento de ellos. Hemos dicho también que es posible esgrimir diferentes defensas dependiendo de la actitud que se adopte frente a cada una de las premisas. Estamos tratando de mostrar que la defensa de Gödel pretende negar la validez de la primera premisa. A la luz de Gödel, hay objetos matemáticos abstractos no espacio-temporales, como hemos tratado de mostrar hasta el momento. La existencia de tales objetos se puede establecer en forma más o menos paralela a la reconstrucción de los objetos físicos en el programa cartesiano. No obstante, tales objetos difieren radicalmente de los objetos físicos y por esa razón tenemos acceso a ellos a través de una intuición diferente de la intuición sensorial. En la siguiente observación de Gödel se ve claramente que tales entidades son diferentes de los objetos físicos:

Sabemos que muchas proposiciones generales acerca de los números naturales son verdaderas (dos más dos es cuatro, hay infinita cantidad de números primos, etc.) y, por ejemplo, creemos que la conjetura de Goldbach tiene sentido, debe ser verdadera o falsa, sin que haya espacio para alguna convención arbitraria. De ahí que debe haber hechos objetivos acerca de los números naturales. Pero estos hechos objetivos deben referir a objetos que deben ser diferentes de los objetos físicos pues, entre otras cosas, son inmodificables en el tiempo. ${ }^{25}$

${ }^{25}$ Citado en Wang 1996, p. 211. 
Ahora bien, si los seres humanos son sólo espacio-temporales, ¿cómo pueden tener ellos intuiciones de objetos no espacio-temporales? Haciendo uso de un chiste de Kripke, ${ }^{26}$ podríamos preguntar: ¿por medio de qué clase de telescopio se puede establecer lo que existe en otro mundo posible? Dado que hemos subrayado algunos rasgos y problemas compartidos con el programa de investigación de Descartes, conviene mencionar también una de las más profundas objeciones formuladas a las Meditaciones metafísicas. Gassendi pregunta en su cuarta objeción contra la sexta meditación: ¿cómo siendo tú inextenso puedes recibir la especie representativa del cuerpo, que es extensa, formada, etc. ${ }^{27}$ Nuevamente, la similitud entre las dificultades de Descartes y las dificultades de Gödel es asombrosa. Descartes tenía que responder a la pregunta: ¿dónde y de qué manera adquiere el alma, que es inextensa, información del mundo exterior que es extenso? Descartes respondió con cierta seguridad: "en la glándula pineal". ${ }^{28}$ A pesar de la seguridad de Descartes, el lector advierte muy pronto la ligereza. Gödel, en sus conversaciones con Hao Wang, ofreció alguna vez una respuesta igualmente insatisfactoria:

Conjeturo que se necesita algún órgano físico para manipular las posibles impresiones abstractas [...]. Tal órgano sensorial debe estar relacionado cercanamente con el centro neural del lenguaje. Pero nosotros por ahora no conocemos lo suficiente y es probable que la teoría primitiva que sobre tales cuestiones tenemos en el presente momento sea comparable al estado de la teoría atómica en los tiempos de Demócrito. ${ }^{29}$

En lo que sigue trataremos de hacer explícito el argumento que le permite a Gödel sostener que los seres humanos no son enteramente espaciotemporales. En este caso nos apoyaremos especialmente en la conferencia que Gödel ofreció en 1951 en una reunión de la American Mathematical Society titulada "Some Basic Theorems on the Foundations of Mathematics and their Implications". ${ }^{30}$ Esta conferencia puede dividirse en dos partes; la primera de ellas también está dividida en dos: una exposición de ciertos resultados lógicos y las consecuencias directas de tales resultados. En la segunda parte de la conferencia, Gödel aduce una serie de consideraciones

${ }^{26}$ Kripke 1980.

${ }^{27}$ Descartes 1641, p. 778.

28 "Hay en el cerebro una pequeña glándula en la cual el alma ejerce sus funciones de un modo más notable que en las demás partes del cerebro" (Descartes 1649, Art. 31, p. 977).

${ }^{29}$ Citado en Wang 1996, p. 233.

${ }^{30} \mathrm{El}$ profesor Rodríguez Consuegra traduce así el título: "Algunos teoremas básicos sobre los fundamentos de la matemática y sus implicaciones filosóficas". El profesor Zalamea ha anotado que tal diferencia sutil en la traducción posee una doble intención: resaltar, por un lado, el aspecto filosófico de la conferencia, y advertir, por el otro, la tesis central del profesor Rodríguez: la primacía del Gödel filosófico sobre el Gödel lógico. Véase Zalamea 1996, p. 362. 
a favor del platonismo. Obviaremos la descripción de los resultados lógicos y nos concentraremos especialmente en las consecuencias.

Los resultados matemáticos que cita Gödel al comienzo de su conferencia están relacionados con la incompletabilidad o la inexhaustibilidad de las matemáticas. Los trabajos lógicos de Gödel han puesto en evidencia dos resultados que sirven de base para las reflexiones siguientes. En primer lugar, para cualquier sistema consistente bien definido de axiomas y reglas de inferencia existen problemas diofánticos ${ }^{31}$ que son indecidibles por esos axiomas y reglas. En un sistema bien definido debe ser posible listar los axiomas en algún formalismo preciso o, en caso de tratarse de un número infinito de axiomas, aportar un procedimiento finito para listarlos uno después del otro. En el caso de las reglas de inferencia, debe ocurrir que dadas cualesquiera premisas, debe ser posible escribir las conclusiones alcanzadas por cada una de las reglas, o se debe poder determinar que no existe ninguna conclusión inmediata a partir de tales reglas. El requisito para los axiomas y para las reglas es equivalente a la condición que se exige para la construcción de una máquina finita en el sentido de Turing. ${ }^{32}$ En segundo lugar, dado que la pregunta por la consistencia de un sistema bien definido de axiomas y reglas de inferencia es una cuestión matemática bien definida, tal cuestión se puede transformar en una cuestión teórica de números (es posible hacer uso de las traducciones gödelianas). Una vez establecida la traducción, es posible mostrar que la proposición que establece la consistencia del sistema (o más bien, la proposición teórica de números equivalente) es indemostrable a partir de tales axiomas y reglas con tal de que tales axiomas y reglas sean consistentes y basten para derivar una determinada porción de la aritmética finitista. Nadie puede construir un sistema formal y afirmar con sentido que él percibe con certeza matemática que los axiomas y reglas son correctos y creer que contiene todas las matemáticas. Si alguien afirma esto, debe reconocer también que percibe la consistencia del sistema. Ahora bien, dado que la consistencia no es demostrable con las herramientas del sistema, esta persona afirma percibir la verdad de algo que no puede ser probado en el mismo y por esa razón debe abandonar la pretensión de que el sistema contenga toda la matemática.

\footnotetext{
${ }^{31}$ Gödel define los problemas diofánticos en los siguientes términos: Sea $P\left(x_{1}, \ldots, x_{n}\right.$, $\left.y_{1}, \ldots, y_{m}\right)$ un polinomio con coeficientes enteros dados y $n+m$ variables tales que las variables $x_{i}$ son desconocidas, y las variables $y_{i}$ operan como parámetros, un problema diofántico se puede plantear en los siguientes términos: ¿tiene la ecuación $P=0$ soluciones enteras para cualesquiera valores enteros de los parámetros, o hay valores enteros de los parámetros para los cuales esta ecuación no tiene soluciones enteras? (SBT, p. 307). En el cursillo dictado en Princeton en 1934, con el ánimo de presentar en forma más detallada los resultados obtenidos acerca de proposiciones indecidibles, Gödel formuló una explicación cuidadosa de la manera como es posible construir equivalentes diofánticos de sentencias indecidibles (véase $U P$, pp. 363-367).

${ }^{32} \mathrm{El}$ teorema mencionado es, entonces, equivalente al hecho de que no existe ningún procedimiento finito para la decisión sistemática de todos los problemas diofánticos (SBT, p. 308).
} 
Todo lo anterior nos obliga a responder la siguiente pregunta con mucha precaución: ¿significa esto que ningún sistema bien definido de axiomas correctos puede contener toda la matemática? Gödel admite que esta pregunta tiene una respuesta doble dependiendo del sentido que demos a la expresión toda la matemática. Es posible hablar de toda la matemática en un sentido objetivo si con ello nos referimos a todas las proposiciones matemáticamente verdaderas en un sentido absoluto. Y es posible hablar de toda la matemática en un sentido subjetivo si con ello nos referimos al sistema de todas las proposiciones matemáticamente demostrables. Si adoptamos el sentido objetivo, debemos responder que, efectivamente, ningún sistema puede contener toda la matemática; el sistema no podría demostrar, por ejemplo, la consistencia del mismo. Si adoptamos el sentido subjetivo no es cierto que ningún sistema contenga toda la matemática, pues nada prohíbe la existencia de una regla finita que genere los axiomas evidentes de la matemática subjetiva. No obstante, ningún sistema finito de reglas podría capturar nuestra intuición matemática, pues, si fuera así, conoceríamos su consistencia y esto va más allá de las reglas. Si esta regla existiese, no podríamos conocerlo en forma absolutamente segura y general; tan sólo podríamos advertir la verdad de una proposición tras otra para un número finito de las mismas. En este caso, tendríamos que admitir que la mente humana equivale a una máquina finita que es incapaz de conocer completamente su funcionamiento. Si la mente humana tuviera las limitaciones de una máquina finita, la matemática objetiva no sólo sería incompletable en el sentido de no estar contenida en ningún sistema axiomático bien definido, sino que habría problemas diofánticos absolutamente irresolubles. Estos problemas no se podrían resolver mediante ninguna prueba que la mente humana pudiese concebir (SBT, p. 310). Esto conduce a Gödel al siguiente dilema:

O la matemática es incompletable en el sentido de que una regla finita no puede nunca abarcar sus axiomas evidentes, es decir, que la mente humana (incluso en el reino de la matemática pura) sobrepasa infinitamente la potencia de cualquier máquina finita, o bien existen problemas diofánticos absolutamente irresolubles. (SBT, p. 310)

Gödel aclara a continuación que nada excluye que las dos alternativas se den simultáneamente. Esta posibilidad constituye entonces una tercera alternativa. La primera alternativa nos lleva a sostener que el funcionamiento de la mente humana no se puede reducir al funcionamiento del cerebro, el cual se puede concebir como una máquina finita con un número finito de partes. La segunda alternativa nos lleva a oponernos radicalmente 
a quienes sostienen que las matemáticas son sólo nuestra creación. ${ }^{33} \mathrm{De}$ nada serviría ofrecer como ejemplo el hecho de que nosotros construimos máquinas mecánicas y no estamos obligados a conocer todos los detalles relacionados con su funcionamiento. La analogía no es completa, pues no construimos nuestros motores a partir de la nada, sino que los elaboramos a partir de un material dado cuya estructura última podemos perfectamente desconocer. Todo esto sugiere que los objetos y hechos matemáticos existen en forma objetiva y son independientes de nuestros actos mentales (STB, p. 311). En otras palabras, la elección es entre: el fisicalismo es falso, el platonismo es verdadero, o el fisicalismo es falso y simultáneamente el platonismo es verdadero. El argumento de Gödel se puede sintetizar de la siguiente manera:

33 Gödel ofrece inicialmente tres argumentos contra una perspectiva constructivista de las proposiciones matemáticas. En primer lugar, si la matemática fuera una libre creación nuestra, podríamos ignorar algunas cuestiones relativas a nuestras creaciones únicamente en virtud de la falta de una descripción completa o en virtud de algunas dificultades prácticas. Tal ignorancia debería disminuir tan pronto como perfeccionamos nuestras construcciones y mejoramos nuestras técnicas. No obstante, las matemáticas modernas han perfeccionado de una manera insospechada las técnicas de construcción y no se han eliminado, ni siquiera disminuido, las cuestiones problemáticas en matemáticas. En segundo lugar, la propia actividad de los matemáticos no exhibe en forma clara la pretendida libertad de creación del matemático: cada teorema nuevo restringe más y más la mencionada libertad. En tercer lugar, si los objetos matemáticos fueran nuestras libres creaciones, los enteros y los conjuntos de enteros serían creaciones completamente diferentes. Sin embargo, necesitamos los segundos si queremos demostrar algunos teoremas básicos en relación con los primeros. A pesar del carácter convincente de los argumentos, Gödel reconoce que están dirigidos hacia un contrincante frágil. Es necesario responder a una versión más fuerte de constructivismo. El contrincante más fuerte que Gödel puede imaginar es, precisamente, el convencionalista. En particular, el convencionalismo de Carnap. En primer lugar, si las proposiciones matemáticas fueran tan sólo expresión de tautologías, sería posible concebir un procedimiento mecánico para decidir acerca de la verdad o falsedad de cada una de ellas. Como este procedimiento no puede existir, habrá que renunciar a la pretendida tautologicidad de las proposiciones matemáticas. En segundo lugar, si cada proposición matemática demostrable se puede deducir a partir de las reglas acerca de la verdad y la falsedad de las proposiciones (sin tener la necesidad de conocer nada acerca de los supuestos objetos que describen), puede ocurrir que tales reglas se constituyan en medios para entender el significado de las fórmulas. Sin embargo, si una teoría tal quiere probar el carácter tautológico de los axiomas, debe empezar por asumir que tales axiomas son verdaderos. Así las cosas, en lugar de definir el significado de tales axiomas por medio de convenciones simbólicas, debemos conocer primero su significado para entender las convenciones sintácticas. En tercer lugar, si queremos probar la consistencia de la teoría clásica de números, debemos hacer uso de ciertos conceptos abstractos, tales como "conjunto", "demostrable", "función de enteros". Estos conceptos no son sintácticos. Hay un sentido en el cual es acertado afirmar que las proposiciones matemáticas carecen de contenido: ellas no enuncian nada acerca de las realidades físicas o psíquicas. Pero no por ello, piensa Gödel, estamos obligados a negar que tales proposiciones describen una realidad objetiva y se reducen a enunciar nuestras creaciones arbitrarias. Las proposiciones matemáticas se ocupan de las relaciones entre conceptos, y los conceptos nos son dados en la intuición matemática en una forma análoga a como los objetos físicos nos son dados en la intuición sensible. 
(1) si nuestra mente pudiese reducirse a un dispositivo espacio-temporal y, en ese sentido, asimilarse a una máquina finita de Turing, sólo podríamos contemplar como verdaderos aquellos teoremas producidos directamente por tal máquina;

(2) nosotros podemos reconocer algunas verdades que no podría producir una máquina de Turing finita que reproduce fielmente un fragmento importante de las verdades que nosotros reconocemos por nuestros medios;

(3) por lo tanto, nuestra mente no puede reducirse a un dispositivo espacio-temporal.

También podemos presentar el argumento así: La mente humana no puede mecanizar —o formalizar - todas sus intuiciones matemáticas. Si tiene éxito al formalizar algunas de ellas, este hecho disparará nuevo conocimiento intuitivo. La mente puede, por ejemplo, advertir la propia consistencia del sistema formal y esto ya no lo puede demostrar con los axiomas previos. En este punto se resume la incompletabilidad de las matemáticas. En otras palabras: si la mente humana es reducible a una máquina de Turing, habrá cuestiones teóricas de la teoría de números que resulten inexorablemente desconocidas para ella. Dado que no hay evidencia de tales cuestiones, se sigue, entonces, que la mente no es reductible a una máquina de Turing. Sin embargo, el argumento no es del todo concluyente. El argumento sólo establece que es muy difícil esperar que la mente humana sea una máquina de Turing. No se descarta de plano tal posibilidad. Gödel era consciente de dicha dificultad:

Los resultados de incompletitud no descartan la posibilidad de que exista un computador que pruebe teoremas y que sea, en efecto, equivalente a la intuición matemática. Pero ellos implican que, en un caso tal, o bien no conocemos la especificación exacta del computador, o bien desconocemos que funciona correctamente. ${ }^{34}$

El argumento de Gödel se puede presentar en términos más generales para captar un nuevo parentesco con el argumento de Descartes. Si la mente fuera un dispositivo mecánico, hay tareas que no podría realizar; nosotros podemos realizar tales tareas; por lo tanto, la mente no es un dispositivo mecánico. En la quinta parte del Discurso del método, Descartes ofreció un argumento para mostrar, por un lado, que el alma no se puede reducir a un mecanismo, y, por otro, que otros seres humanos diferentes de mí poseen también un alma. En primer lugar, Descartes plantea que se pueden

34 Citado en Wang 1996, p. 186. 
explicar todos los aspectos de la conducta animal partiendo de la hipótesis de que el animal es un autómata. Descartes era un experto en fisiología especulativa. También podemos explicar el funcionamiento de una máquina que produzca un número bastante elevado de proposiciones verdaderas de la teoría de números. Descartes sostuvo después que el hombre posee ciertas facultades que no se pueden explicar por medios mecánicos, aunque buena parte de las conductas del hombre sí se puedan explicar así. Es precisamente el lenguaje humano el que exhibe la diferencia más grande entre el animal y el hombre; o entre un mecanismo sofisticado y el hombre. Deseo citar in extenso el argumento de Descartes:

En este lugar de mi tratado me detuve de forma particular en hacer ver que, si hubiese máquinas tales que tuviesen los órganos y la figura de un mono o cualquier otro animal sin razón, no tendríamos medio alguno para reconocer que tales máquinas no tuviesen la misma naturaleza que estos animales. Por el contrario, si hubiese alguna que tuviese semejanza con nuestro cuerpo e imitase todas nuestras acciones tanto como moralmente fuese posible, tendríamos siempre medios muy seguros para conocer que no serían verdaderos hombres. El primero es que nunca podrían usar palabras, ni componer otros signos, como nosotros hacemos, para manifestar a otros nuestros pensamientos. Pues puede fácilmente pensarse que una máquina esté hecha de forma tal que profiera palabras e incluso que profiera algunas a propósito de acciones corporales que causasen cierto cambio en sus órganos, como si se pulsa en un determinado lugar que pregunte qué se le quiere decir, y si se le pulsa en otro que grite que se le hace daño, y cosas semejantes; pero no será capaz de utilizar de forma diversa las palabras para responder con sentido a todo cuanto se diga en su presencia, tal y como los hombres menos capacitados pueden realizar. El segundo es que aunque fuesen capaces de ejecutar varias acciones tan correctamente o mejor que ninguno de nosotros, fallarían sin duda en otras, en virtud de lo cual descubriríamos que no obran por conocimiento, sino solamente en virtud de la disposición de sus órganos. Pues, así como la razón es un instrumento universal, capaz de servir en cualquier circunstancia, estos órganos, por el contrario, exigen una disposición particular para cada acción concreta. Por lo cual es moralmente imposible que exista una máquina con tal variedad de resortes dispuestos en una forma tal que pudieran hacerla actuar en todas las coyunturas de la vida, tal y como nos hace actuar nuestra razón. ${ }^{35}$

Descartes está pensando en máquinas compuestas de resortes y animadas por circuitos hidráulicos; Gödel está pensando en máquinas de Turing instanciadas, quizá, en circuitos eléctricos. Descartes cree que una máquina con resortes no podría hablar con sentido en la forma como nosotros lo hacemos; Gödel cree que una máquina de Turing no podría advertir la consistencia de la teoría de números, o su propia consistencia en la forma como nosotros lo hacemos. Las máquinas, cree Descartes, no podrían

35 Descartes 1637, pp. 628-629. 
usar el lenguaje como nosotros lo hacemos. Las máquinas, cree Gödel, no podrían hacer matemáticas como nosotros lo hacemos.

A juzgar por la estructura del argumento de Benacerraf, la defensa del platonismo, contrario a lo que podría esperar Gödel, exige que las dos alternativas avizoradas en la conferencia Gibbs sean correctas: el fisicalismo es inadecuado y el realismo de los objetos abstractos es correcto. La segunda parte de la disyunción señalada por Gödel coincide con la segunda premisa de Benacerraf. Por lo tanto, no es posible debilitar el argumento de Benacerraf si, en forma simultánea, no ocurre que la primera parte de la disyunción de Gödel niegue la fortaleza de la primera premisa de Benacerraf. En otras palabras, no basta con sugerir que los objetos matemáticos no son creación nuestra y existen por fuera del espacio-tiempo. También es necesario mostrar las limitaciones del fisicalismo y dejar claro que la mente no es reductible a alguna explicación espacio-temporal. Es precisamente este segundo aspecto el que falla en la defensa de Gödel. Sus argumentos, tal como lo reconoce él mismo, sólo establecen la posibilidad de que una máquina que prueba teoremas no pueda captar en forma completa la intuición matemática; no demuestran esto con absoluta seguridad. Tan consciente era Gödel de la debilidad de su argumento contra el fisicalismo, que en ocasiones optó por ofrecer argumentos empíricos. Gödel creía que se podría demostrar que el tiempo necesario para que fuera posible la formación, a partir de las leyes de la física, de seres vivos que de alguna manera replican las características esenciales de un ser humano, era comparable al tiempo que deberíamos esperar para que, por simple azar, se separaran los componentes de la atmósfera terrestre. ${ }^{36}$

La idea del argumento central de Gödel, presentado en la conferencia Gibbs, es la siguiente:

(i) hay tareas que una máquina, en particular una máquina de Turing, no puede realizar: la máquina no puede demostrar su propia consistencia;

(ii) nosotros podemos adelantar tales tareas: podemos reconocer la consistencia de un sistema porque podemos explorar por fuera de él —contamos con intuición matemática一;

(iii) por lo tanto, no somos máquinas.

La debilidad del argumento reside en que no logra mostrar de una manera indubitable la fuerza de la primera premisa. En 1961, diez años más tarde, J.R. Lucas propuso un argumento similar que se puede resumir en los siguientes términos: una máquina de Turing no podría tener información

\footnotetext{
${ }^{36}$ Véase en Wang 1996, p. 192.
} 
acerca de los fenómenos de incompletitud. ${ }^{37} \mathrm{~A}$ una máquina se le podría anexar otro dispositivo que produjera una fórmula que la primera máquina no pudiese producir. No obstante, esta última sería una máquina diferente de la inicial. Dado que la mente es consciente de los fenómenos de incompletitud sin que sea necesario anexar un dispositivo que la convierta en algo diferente, debemos concluir que las mentes no son máquinas de Turing. Un ser consciente, según Lucas, puede serlo de sí mismo y de otras cosas sin que se le tenga que analizar como divisible en partes. La clave del argumento reside en la siguiente creencia: no es posible construir una máquina que considere su propia actuación sin convertirla en una máquina diferente, es decir, sin agregar un dispositivo que identifique fórmulas gödelianas en la matriz original. Hilary Putnam se ha encargado de producir una serie de ingeniosas alternativas para responder a la familia de argumentos gödelianos, incluido el intento de Lucas. En el artículo "Minds and Machines", el autor sugiere que la posibilidad de construir un sistema de computación capaz de responder preguntas sobre su propia estructura no puede descartarse por argumentos lógicos y, en consecuencia, esa particular propiedad no tiene nada que ver con una supuesta naturaleza única de la experiencia subjetiva humana. ${ }^{38}$ Putnam trata de mostrar que la pregunta: "icómo puede una máquina verificar que se encuentra en un estado particular?" es tan extraña como la pregunta: "icómo puede usted verificar que le duele una muela?" Putnam se refiere a esta clase de preguntas con el término: preguntas desviadas. Para defender la posible analogía entre una máquina y un ser humano, Putnam propone distinguir entre estados lógicos y estados estructurales. Los primeros se describen usando el vocabulario de las máquinas de Turing, los segundos se describen usando las características físicas de los dispositivos que instancian una máquina de Turing. En ese orden de ideas, tan pronto como construimos físicamente una máquina de Turing, ella no dispone de medios para verificar sus estados estructurales de la misma manera en que un ser humano tampoco dispone de los medios para verificar el estado de su apéndice en un momento dado. El análisis de Putnam no muestra que efectivamente las mentes son máquinas, pero deja ver con claridad, como el mismo Gödel ya lo había advertido, que la familia de argumentos gödelianos no es concluyente. Putnam termina su artículo advirtiendo que o bien ningún argumento de los filósofos arroja una luz clara acerca de la relación mente-cuerpo, o bien una buena parte del mecanicismo es correcto, o, en caso de que algún argumento dualista demuestre que los seres humanos son una mezcla de cuerpo y alma, ese argumento mostrará también que las máquinas de Turing son una mezcla de circuitos integrados y alma. A pesar de las buenas intenciones del argumento de Gödel, debemos advertir que no es del todo concluyente.

${ }^{37}$ Lucas 1961.

${ }^{38}$ Putnam 1960. 
En síntesis: podemos argumentar, siguiendo a Gödel, que adquirimos conocimiento de los objetos matemáticos abstractos de la misma manera como adquirimos el conocimiento de los objetos físicos concretos. Conocemos los objetos físicos por medio de la percepción sensorial y los objetos abstractos por medio de la intuición matemática. Si pudiéramos sostener que las mentes humanas son ellas mismas inmateriales y no espacio-temporales, podríamos ser capaces de construir una visión de acuerdo con la cual adquiriríamos información acerca de los objetos matemáticos aun cuando no hubiese señales que pasen del reino matemático al reino espaciotemporal. No obstante, hemos de concluir que el argumento de Gödel no resuelve definitivamente el aspecto no espacio-temporal de la mente y, en consecuencia, todavía no es posible estructurar, a partir de él, una defensa completa del platonismo.

\section{BIBLIOGRAFÍA}

\section{Convenciones para citar la obra de Gödel}

CCP, 1947/1964, "What is Cantor's Continuum Problem?", en CWII, pp. 176-188, 254-270. Existe una versión en español en OC, pp. 354-370; 424-429.

CWI, 1986, Kurt Gödel: Collected Works, vol. I (publicaciones de 1929-1936), ed. Solomon Feferman, John W. Dawson, Jr., Stephen C. Kleene, Gregory H. Moore, Robert M. Solovay y Jean Van Heijenoort, Oxford University Press/ Clarendon Press, Nueva York/Oxford.

CWII, 1990, Kurt Gödel: Collected Works, vol. II (publicaciones de 1938-1974), ed. Solomon Feferman, John W. Dawson, Jr., Stephen C. Kleene, Gregory H. Moore, Robert M. Solovay y Jean Van Heijenoort, Oxford University Press, Nueva York.

CWIII, 1995, Kurt Gödel: Collected Works, vol. III (ensayos y conferencias inéditas), ed. Solomon Feferman, John W. Dawson, Jr., Warren Goldfarb, Charles Parsons y Robert N. Solovay, Oxford University Press, Nueva York.

DFM, 1961, "The Modern Development of the Foundations of Mathematics in the Light of Philosophy", en CWIII, pp. 374-387.

OC, 1989, Kurt Gödel: Obras completas, ed. y trad. Jesús Mosterín, Alianza, Madrid.

$R M L, 1944$, "Russell's Mathematical Logic", en CWII, pp. 119-141. Existe una versión en español en OC, pp. 313-343.

SBT, 1951, "Some Basic Theorems on the Foundations of Mathematics and their Implications", en CWIII, pp. 304-323. Existe una versión en español en Rodríguez Consuegra 1994, pp. 149-187.

TRKP , 1946/1949, "Some Observations about the Relationship between Theory of Relativity and Kantian Philosophy", en CWIII, pp. 230-259.

UP, 1934, "On Undecidable Propositions of Formal Mathematical Systems", en CWI, pp. 346-371. 


\section{Otras fuentes}

Anderson, Alan Ross (comp.), 1964, Minds and Machines, Prentice Hall, Englewood Cliffs, NJ.

Balaguer, Mark, 1998, Platonism and Anti-Platonism in Mathematics, Oxford University Press, Oxford.

Benacerraf, Paul, 1973, "Mathematical Truth", en Benacerraf y Putnam 1983, pp. 403-420.

Benacerraf, Paul y Hilary Putnam (comps.), 1983, Philosophy of Mathematics, Cambridge University Press, Cambridge, GB.

Descartes, René, 1996, OEuvres Philosophiques, ed. F. Alquié, Classiques Garnier, París.

—_, 1637, Discours de la méthode, en Descartes 1996, vol. I.

_-, 1641, Les Méditations, les objections et les responses, en Descartes 1996, vol. II.

—_, 1649, Les Passions de l'âme, en Descartes 1996, vol. III.

Detlefsen, Michael, 1990, "On an Alleged Refutation of Hilbert's Program Using Gödel's First Incompleteness Theorem", en Journal of Philosophical Logic, vol. 19, no. 4, pp. 343-347.

Feferman, Solomon, 1988, "Kurt Gödel: Conviction and Caution", en Shanker 1988, pp. 96-114.

Frege, Gottlob, 1980, Philosophical and Mathematical Correspondence, The University of Chicago Press, Chicago.

— , 1950, The Foundations of Arithmetic, Northwestern University Press, Evanston, Illinois.

Galilei, Galileo, 1984, El mensaje y el mensajero sideral, trad. Carlos Solís Santos, Alianza, Madrid.

Hintikka, Jaako, 1998, "On Gödel's Philosophical Assumptions”, Synthèse, vol. 114, pp. 13-23.

Kripke, Saul, 1980, Naming and Necessity, Harvard University Press, Cambridge, Mass. [versión en castellano: El nombrar y la necesidad, 2a. ed., trad. Margarita M. Valdés, Instituto de Investigaciones Filosóficas-UNAM, 1995].

Lucas, J.R., 1961, "Minds, Machines and Gödel”, Philosophy, vol. 36, pp. 120-124.

Maddy, Penelope, 1990, Realism in Mathematics, Oxford University Press, Oxford.

Putnam, Hilary, 1960, "Minds and Machines", en Anderson 1964, pp. 43-59.

Rodríguez Consuegra, Francisco (comp.), 1994, Ensayos inéditos de Kurt Gödel, Biblioteca Mondadori, Barcelona.

Shanker, S.G. (comp.), 1988, Gödel's Theorem in Focus, Routledge, Londres.

Wang, Hao, 1996, A Logical Journey, from Gödel to Philosophy, The MIT Press, Cambridge, Mass.

—_ 1987, Reflexiones sobre Kurt Gödel, trad. Pilar Castillo Criado, Alianza, Madrid.

—_, 1981, "Some Facts about K. Gödel", Journal of Symbolic Logic, vol. 46, pp. 653-659.

— 1974, From Mathematics to Philosophy, Routledge and Kegan Paul, Londres.

Zalamea, Fernando, 1996, "Kurt Gödel: análisis filosófico y lógica matemática", Mathesis (México), vol. 12, pp. 347-374.

Recibido el 11 de enero de 2002; aceptado el 30 de abril de 2002 